DOI: $10.5216 /$ rpp.v14i2.10269

\title{
O TREINO DAS TÉCNICAS AVANÇADAS DE TENISTAS ADULTOS FEDERADOS: UM ESTUDO COMPARATIVO ENTRE TRÊS DIFERENTES CATEGORIAS
}

\author{
Marcelo Meirelles da Motta
}

Universidade do Vale do Rio dos Sinos, Porto Alegre, Rio Grande do Sul, Brasil

Marcos Alencar Abaide Balbinotti

Universidade do Vale do Rio dos Sinos, Porto Alegre, Rio Grande do Sul, Brasil

Marcus Levi Lopes Barbosa

Universidade Federal do Rio Grande do Sul, Porto Alegre, Rio Grande do Sul, Brasil

Ricardo Pedrozo Saldanha

Universidade Federal do Rio Grande do Sul, Porto Alegre, Rio Grande do Sul, Brasil

\section{Roberto Tierling Klering}

Universidade Federal do Rio Grande do Sul, Porto Alegre, Rio Grande do Sul, Brasil

\section{Carlos Adelar Abaide Balbinotti}

Universidade Federal do Rio Grande do Sul, Porto Alegre, Rio Grande do Sul, Brasil

Resumo: $\mathrm{O}$ treino das técnicas avançadas no tênis consiste no exercício sistemático dos Golpes de Preparação (GP) e dos Golpes de Definição (GD). Trata-se de combinações de golpes executados durante as competições. O objetivo desse estudo é responder duas questões norteadoras: (1) Qual a freqüência de treinamento dos Golpes de Preparação e dos Golpes de Definição de tenistas adultos federados; (2) Há diferenças estatisticamente significativas na comparação dos resultados das três categorias oficiais estabelecidas pela Federação Gaúcha de Tênis (FGT): "Livre, Maiores A, Maiores B"? Para tanto, foi aplicado o Inventário do Treino Técnico-desportivo do Tenista (ITTT12), no qual os tenistas respondem com que freqüência eles treinam as 12 principais ações que constituem as técnicas avançadas no tênis. Através dos resultados obtidos, foi possível concluir que os GP são, indiscutivelmente, as ações mais relevantes na performance dos tenistas adultos federados.

Palavras-chave: Tênis. Técnicas Avançadas. Tenistas Adultos.

\section{Introdução}

O tema central desse estudo trata de conteúdos específicos do treinamento das técnicas avançadas no tênis. Mais especificamente, apresenta resultados sobre a freqüência de treinamento dos Golpes de Preparação (GP) e dos Golpes de Definição 
(GD) de tenistas adultos federados, a partir das respostas obtidas pelo Inventário do Treino Técnico-desportivo de Tenistas (ITTT-12). Esse instrumento é constituído por 12 jogadas - seis GP e seis GD -, que compõem as principais ações técnico-desportivas do tenista em situação de jogo. Além dos resultados obtidos de uma amostra geral de 99 tenistas, ainda foi realizado um estudo comparativo entre três diferentes categorias federadas: "Livre, Maiores A, e Maiores B".

Técnicas avançadas no tênis: pressupostos teóricos

Para o aperfeiçoamento das técnicas avançadas dos tenistas é recomendável o exercício sistemático e equilibrado das 12 combinações de golpes, principalmente, em situação de treino (BALBINOTTI, 2003a; BALBINOTTI; MOTTA, 2009a). Isso porque na situação de competição, o tenista deverá, além de tentar impor suas potencialidades técnicas diante de seu adversário, também se espera dele a capacidade de responder as ações desse adversário contra suas deficiências técnicas (BALBINOTTI; MOTTA, 2009b; CRESPO; MILEY, 1999; WOODS; FERNANDES, 2001; WOODS; HOCTOR; DESMOND, 1995). O princípio fundamental do treino das técnicas avançadas no tênis é a necessidade de desenvolver um grupo diversificado e multilateral de ações motoras, que permitam inúmeras variações de golpes (DENIAU, 1991; GROPPEL, 1993; SCHÖMBORN, 1999). Mesmo que o tenista apresente maior competência na realização de algumas ações em comparação a outras, é preciso que o treino seja orientado de forma proporcional, não permitindo o treinamento de algumas das técnicas avançadas em detrimento de outras. Caso contrário, em médio e longo prazo as limitações decorrentes desse processo poderão se tornar grandes deficiências na perspectiva do alto rendimento do tenista (BALBINOTTI, 2003b; WOODS; FERNANDES, 2001; WOODS; HOCTOR; DESMOND, 1995).

Portanto, o erro comum que deve ser evitado pelo treinador ocorre quando o treino das técnicas avançadas é orientado de forma unilateral, ou seja, para um grupo específico de jogadas em detrimento de uma ampla variedade de ações (BALBINOTTI; MOTTA, 2009a; DENIAU, 1991; GROPPEL, 1993). É preciso estar atento a isso em razão do fato de que o tenista, por sua conta, possui a tendência de praticar as ações motoras que lhe trazem maior retorno positivo e, por isso, evitam àquelas que resultam em erros e desprazer. É perfeitamente compreensível que o tenista procure utilizar suas jogadas mais eficientes, inclusive, em situação de treino e competições simuladas, visando adquirir mais confiança no desempenho; no entanto, à medida que vai evoluindo a qualidade dos adversários, maior será a dificuldade de mascarar as reais deficiências. Isso poderá comprometer o projeto do tenista vencedor nas competições (ZLESAK, 1994a; 1994b).

Modelo de avaliação do treino das técnicas avançadas no tênis

Tendo como base os pressupostos teóricos das técnicas avançadas no tênis, Balbinotti e Balbinotti (2003) elaboraram o Inventário do Treino Técnico-desportivo do Tenista (ITTT-12), no qual os tenistas respondem com que freqüência eles treinam as 12

Pensar a Prática, Goiânia, v. 14, n. 2, p. 1-15, maio/ago. 2011 
principais ações que constituem as técnicas avançadas no tênis. Essas 12 ações, divididas em duas categorias - GP e GD -, contemplam diferentes variações da técnica padrão do tenista: movimentações laterais (ex: troca de bolas de fundo de quadra); movimentações verticais (ex: saque com aproximação à rede); potência (ex: saque como golpe vencedor); mudança de rotação e altura (ex: troca de bolas com variações de potência e rotação); entre outras. O ITTT-12 é um instrumento construído e validado no estudo de Balbinotti (2003), que foi utilizado em outras pesquisas, tanto com tenistas juvenis quanto adultos: (BALBINOTTI et al., 2003; BALBINOTTI et al., 2004a; BALBINOTTI et al., 2005).

Questões centrais da investigação

Dada a relevância do controle do treinamento das técnicas avançadas no tênis, o estudo propõe duas questões norteadoras: (1) Qual a freqüência de treinamento dos Golpes de Preparação e dos Golpes de Definição de tenistas adultos federados; (2) Há diferenças estatisticamente significativas na comparação dos resultados das três categorias oficiais estabelecidas pela Federação Gaúcha de Tênis (FGT): "Livre, Maiores A, Maiores B'?

\section{Material e métodos}

Caracterização do Estudo

Trata-se de um estudo descritivo-exploratório, que pretende examinar comparações entre variáveis, bem como gerar predições (THOMAS; NELSON, 2002).

População e Amostra

Para esta pesquisa foi utilizada uma amostra de 99 tenistas adultos do sexo masculino, pertencentes às seguintes categorias oficiais das competições organizadas pela Federação Gaúcha de Tênis (FGT): 1) "Livre": categoria composta por tenistas de melhor desempenho amador, não havendo limite de idade; 2) "Maiores A": categoria amadora adulta composta em sua maioria por tenistas de desempenho intermediário, limitado a atletas acima de 19 anos; 3) "Maiores B": categoria amadora adulta que compreende, em sua maioria, tenistas iniciantes à modalidade e que apresentam técnica rudimentar, também limitado a atletas acima de 19 anos e que não ocupem uma posição inferior ao décimo lugar na categoria "Maiores A". Todos os tenistas participam regularmente das competições do circuito regional de tênis e foram escolhidos pelos critérios de disponibilidade e acessibilidade (MAGUIRE; ROGERS, 1989). O estudo conta com uma amostra de 33 indivíduos para cada categoria analisada.

Pensar a Prática, Goiânia, v. 14, n. 2, p. 1-15, maio/ago. 2011 
Instrumento

Para esta pesquisa foi utilizado o Inventário do Treino Técnico-desportivo do Tenista (ITTT-12), que consta de 12 itens que pretendem verificar duas dimensões associadas ao treino técnico-desportivo do tenista - Golpes de Preparação (GP); e Golpes de Definição (GD). As respostas aos itens do ITTT-12 foram dadas conforme uma escala de tipo Likert, bidirecional graduada em cinco pontos, indo de "pouquíssima freqüência" (1) a "muitíssima freqüência" (5). O ITTT-12 foi construído e validado (BALBINOTTI, 2003; BALBINOTTI, et. al., 2004b) conforme os princípios comumente aceitos na literatura internacional (ANGERS, 1992; ASÇI; ASÇI; ZORBA, 1999; BARTOLOMEW, et. al., 1998; EKLUND; WHITEHEAD; WELK, 1997; BISQUERA, 1987). Nestes estudos foram apresentados detalhadamente, os seguintes resultados do processo de proposição e validação do ITTT-12: análises de itens, matriz de correlação entre os itens de cada uma das duas sub-escalas, estrutura fatorial exploratória, e validação de conteúdo dos itens.

No cabeçalho deste instrumento estão presentes algumas questões que tem por objetivo trazer informações sócio-demográficas dos participantes da pesquisa, como: nome, categoria em que disputa, tempo de participação em competições, ranking oficial e tempo de prática semanal.

Procedimentos

Inicialmente, o projeto de pesquisa foi submetido à análise do Comitê de Ética e Pesquisa da Universidade Federal do Rio Grande do Sul (UFRGS) e aprovado na reunião n ${ }^{0} 39$, ata $\mathrm{n}^{0} 119$ de 20 de novembro de 2008 sob o número 2007961.

Após sua aprovação, procedeu-se a aplicação do ITTT-12 durante o intervalo das competições da FGT, que ocorreram entre os meses de Janeiro e Março de 2009. Não houve exclusões, a aplicação ocorreu com todos os atletas que demonstraram interesse em responder o ITTT-12; os dados dos tenistas que não satisfizeram os critérios de seleção não foram compilados. Conforme acordado com os atletas, os nomes dos participantes que responderam o ITTT-12 não serão revelados.

Quanto aos procedimentos estatísticos, o banco de dados foi construído no pacote estatístico SPSS 15.0. Os dados obtidos foram analisados conforme as etapas a seguir. Primeiramente, foram calculados os índices que sustentaram a aderência à normalidade dos dados ( $\mathrm{S}-\mathrm{W}$ com correção Lilliefors, Assimetria, Achatamento). Foram calculados também os índices de tendência central, não-central e dispersão. Por fím, foram comparadas as médias através de Testes $t$ de Student e Anova com seus respectivos testes complementares.

\section{Apresentação dos resultados}

A fim de responder adequadamente a questão central desta pesquisa, realizou-se a exploração dos escores obtidos pelo ITTT-12, seguindo princípios largamente aceitos e descritos na literatura especializada (BISQUERA, 1987; BRYMAN; CRAMER, 1999;

Pensar a Prática, Goiânia, v. 14, n. 2, p. 1-15, maio/ago. 2011 
PESTANA; GAGEIRO, 2003; REIS, 2000; SIRKIN, 1999). Cumprida esta etapa, segue-se a apresentação das estatísticas descritivas e, das comparações das médias entre categorias.

\section{Estatísticas descritivas}

Quanto às três categorias em análise, os resultados relativos às médias de freqüência do treinamento (das variáveis GP e GD) apresentam variações consideráveis $(14,81$ a 22,15) independente da categoria (ver Tabela 1). Considerando os valores nominais, ambos os golpes (GP e GD) são mais praticados pelas categorias "Livre" e "Maiores A", de maneira que as menores freqüências de treinamento ocorreram na categoria "Maiores B".

Estas três categorias apresentaram medianas com valores nominais aproximados das médias (ver Tabela 1). As médias aparadas a 5\% para as três categorias apresentaram valores nominais muito próximos às médias das dimensões GD e GP. Este fato sugere que os casos extremos também não estão afetando as médias das dimensões. As categorias "Livre" e "Maiores A" apresentaram Múltiplas Modas para a dimensão GD, sendo que a categoria "Maiores A" também apresentou Múltiplas Modas para a dimensão GP. Estes fatos podem sugerir problemas referentes à normalidade das dimensões. Todos os outros grupos apresentaram apenas uma Moda.

Tabela 1: Estatísticas de Tendência Central, de Dispersão e Distribuição da amostra com as variáveis "categoria" e "ranking" controladas.

\begin{tabular}{|c|c|c|c|c|c|c|c|c|c|c|c|}
\hline \multirow[b]{2}{*}{ Dimensões } & \multirow[b]{2}{*}{ Categorias } & \multicolumn{5}{|c|}{ Tendência Central e Não Central } & \multicolumn{3}{|c|}{ Normalidade } & \multirow{2}{*}{$\begin{array}{c}\text { Assimetria } \\
\text { Skewness/EP }\end{array}$} & \multirow{2}{*}{$\begin{array}{l}\text { Achatamento } \\
\text { Kurtosis/EP }\end{array}$} \\
\hline & & $\chi_{(D P)}$ & $\begin{array}{l}\text { Mínimo/ } \\
\text { Máximo }\end{array}$ & Mediana & $\begin{array}{c}\chi \\
\text { aparada } \\
\mathbf{5 \%} \\
\end{array}$ & Moda & S-W & gl & sig & & \\
\hline \multirow{3}{*}{ Golpes de Definição } & Livre & $16,24_{(3,57)}$ & $11-25$ & 15 & 16,04 & $14,00^{1}$ & 0,91 & 33 & 0,01 & 2,28 & 0,27 \\
\hline & Maiores A & $16,57_{(2,81)}$ & $12-23$ & 17 & 16,51 & $16,00^{2}$ & 0,96 & 33 & 0,28 & 0,06 & $-0,7$ \\
\hline & Maiores B & $14,81_{(3,13)}$ & $08-22$ & 14 & 14,81 & 14,00 & 0,97 & 33 & 0,38 & $-0,25$ & $-0,41$ \\
\hline \multirow{3}{*}{ Golpes de Preparação } & Livre & $22,15_{(3,37)}$ & $17-30$ & 22 & 22,04 & 20,00 & 0,95 & 33 & 0,15 & 0,65 & $-0,88$ \\
\hline & Maiores A & $21,57_{(3,43)}$ & $15-28$ & 21 & 21,56 & $19,00^{3}$ & 0,96 & 33 & 0,25 & 0,5 & $-1,11$ \\
\hline & Maiores B & $18,36_{(3,71)}$ & $11-27$ & 18 & 18,27 & 19,00 & 0,97 & 33 & 0,52 & 1,04 & 0,17 \\
\hline
\end{tabular}

Obs: 1) Há múltiplas modas, os menores valores encontram-se na tabela ${ }^{1}(15) ;{ }^{2}(19) ;{ }^{3}(23)$. 2) O nível de significância adotado foi de 0,05. 3) gl - Grau de liberdade 4) sig - Significância 5) S-W - Shapiro Wilk.

No que se refere à dispersão da amostra, percebe-se que aqui também não houve uma grande variação entre o desvio-padrão das três categorias, na relação com as dimensões GD e GP. Destaca-se novamente que em nenhuma categoria o desvio-padrão ultrapassou a metade do valor nominal das médias, indicando que a variabilidade e a dispersão dos dados estão satisfatórias. Dos valores referentes às estatísticas de dispersão (ver Tabela 1), nota-se que a menor amplitude total ocorreu na categoria "Maiores A" relacionada à dimensão GD (11); enquanto que a maior ocorreu na categoria "Maiores B” relacionada com a dimensão GP (16).

Pensar a Prática, Goiânia, v. 14, n. 2, p. 1-15, maio/ago. 2011 
Quanto aos valores máximos, observa-se que para as dimensões GD (de 22 a 25 pontos) e GP (de 27 a 30 pontos), a variabilidade é relativamente baixa, considerando o valor nominal expresso. Quanto aos valores mínimos, a variabilidade também é relativamente baixa: GD (de 8 a 11 pontos) e GP (de 11 a 17 pontos), considerando o valor nominal expresso. A baixa variabilidade encontrada ( 3 a 6 pontos), tanto nos valores máximos quanto nos valores mínimos, indica certa homogeneidade nos casos em ambos os extremos da curva.

Quanto aos índices de normalidade da distribuição da amostra, foram testados através do cálculo Shapiro-Wilk $(\mathrm{SW})$. Apenas a categoria "Livre" relacionada à dimensão GD, a aderência à normalidade $(p<0,05)$ não pode ser assumida. Em todas as outras situações entre categorias e dimensões houve aderência à normalidade $(p>0,05)$. Já a análise da assimetria $(-1,96<$ Skewness/EPs $<1,96)$ e achatamento $(-1,96<$ Kurtosis/EPk < 1,96) das distribuições indicam que as curvas se apresentam simétricas e mesocúrticas para ambos os grupos de análise, GD e GP, e as três categorias.

Comparações de médias

Inicialmente, comparações entre dimensões foram realizadas com o auxilio do teste $t$ pareado. Optou-se por este teste por se ter assumido que as distribuições aderem à normalidade em todos os níveis das variáveis controladas (ver Tabela 1) e porque a única exceção a este critério tem em seu grupo mais de 30 sujeitos, o que dispensa o critério da normalidade para a análise (PESTANA; GAGEIRO, 2003). Como se pode ver na Tabela 2 diferenças altamente significativas $(\mathrm{p}<0,01)$ entre as dimensões GP e GD foram encontradas tanto para a mostra geral quanto para cada grupo e subgrupo da amostra, sempre favoráveis à dimensão GP.

Tabela 2: Comparações entre as dimensões Golpes de Preparação e Golpes de Definição nas categorias.

\begin{tabular}{lccc}
\hline Categoria & Teste $\boldsymbol{t}$ & $\begin{array}{c}\text { Grau de } \\
\text { liberdade }\end{array}$ & Significância \\
\hline Livre & 8,288 & 33 & 0,000 \\
Maiores A & 6,277 & 33 & 0,000 \\
Maiores B & 5,689 & 33 & 0,000 \\
\hline
\end{tabular}

Uma vez realizadas as comparações entre as dimensões, cabe realizar as comparações entre categorias, em cada dimensão. Levando em conta que foi assumida a homogeneidade das variâncias $\left(F_{\text {Levene }(2,96)}>0,014 ; \mathrm{p}>0,547\right)$ para as duas dimensões, optou-se por utilizar o teste ANOVA One-way a fim de verificar as diferenças entre os escores em cada categoria. A realização do teste ANOVA revelou haver ao menos uma diferença significativa na dimensão GP, entre as categorias (ver Tabela 3).

Pensar a Prática, Goiânia, v. 14, n. 2, p. 1-15, maio/ago. 2011 
Tabela 3: Teste Anova intradimensões para categorias.

\begin{tabular}{lccc}
\hline Dimensão & Teste $\boldsymbol{F}$ & $\begin{array}{c}\text { Grau de } \\
\text { Liberdade }\end{array}$ & Significância \\
\hline Golpe de Definição & 2,820 & 2 & 0,065 \\
Golpe de Preparação & 11,148 & 2 & 0,000 \\
\hline
\end{tabular}

A fim de localizar em quais categorias especificamente há esta diferença, foi realizado um teste "Tukey B" (já que a homogeneidade da variância foi assumida). Como era esperado, o teste "Tukey B" revelou que para a dimensão GD, as três categorias ("Livre", "Maiores A" e "Maiores B") não apresentavam diferenças estatisticamente significativas ( $\mathrm{p}>0,05)$ entre si. Já para a dimensão GP, o teste "Tukey B" revelou que as categorias "Livre" e "Maiores A" são estatisticamente indissociáveis $(p>0,05)$ e significativamente diferentes $(p<0,05)$ em sua freqüência de treino da categoria "Maiores B" (ver Tabela 4).

Tabela 4: Apresentação dos resultados do Teste Tukey B.

\begin{tabular}{llcc}
\hline \multirow{2}{*}{ Dimensão } & Categoria & \multicolumn{2}{c}{ Agrupamentos $(\mathbf{p}<, \mathbf{0 5})$} \\
\hline \multirow{3}{*}{ Definição } & Maiores B & 14,81 & $\mathbf{2}$ \\
& Livre & 16,24 & -- \\
& Maiores A & 16,57 & -- \\
& & & \\
\multirow{3}{*}{ Preparação } & Maiores B & -- & 18,36 \\
& Maiores A & 21,57 & -- \\
& Livre & 22,15 & -- \\
\cline { 2 - 4 } & & &
\end{tabular}

\section{Discussão dos resultados}

Os resultados apresentados possibilitam uma série interessante de constatações e discussões a respeito do tema central deste estudo. Alguns dados aproximam-se de fundamentações trazidas da literatura. Mas a maior contribuição se revelou em alguns resultados até certo ponto surpreendentes sobre a freqüência de treinamento dos GP e GD.

Os resultados referentes às médias obtidas para a amostra geral demonstram que os tenistas praticam com maior freqüência os GP em comparação aos GD. Estudos anteriores (BALBINOTTI et. al, 2008; BALBINOTTI et. al, 2004a) já apresentaram resultados semelhantes para tenistas infanto-juvenis. A amostra se revelou, na sua totalidade, ser composta de tenistas não-iniciantes em competições, pois se trata de tenistas que competem desde a adolescência.

Nessa perspectiva, a prática do tênis acontece fundamentalmente através da troca de bolas de fundo de quadra. A regularidade e a possibilidade de colocar o adversário em situação extrema de dificuldades que resultem em erros valem mais do que o risco decorrente da tentativa de finalização do ponto (BALBINOTTI; MOTTA, 2009b; WOODS; FERNANDES, 2001; CRESPO; MILEY, 1999; WOODS; HOCTOR; 
DESMOND, 1995). Isso porque este risco proveniente da tentativa da definição apressada da situação normalmente faz crescer significativamente a chance do erro, pois este tipo de jogada necessita de potencia e precisão exacerbada.

A máxima postulada de que os tenistas devem procurar executar técnicas capazes de manter a bola na quadra, independente da situação em que se encontrem, forçando o adversário a efetuar ao menos mais um golpe, costumeiramente esteve e está presente sempre que se leva em consideração informações sobre o jogo fornecidas por parte dos treinadores (DELFORGE, 2006; WOODS; FERNANDES, 2001; WOODS; HOCTOR; DESMOND, 1995; GROPPEL, 1993). Porém, o objetivo de manter a bola em jogo não pode apenas se restringir em esperar o erro simples do adversário. Muitas vezes o tenista se vê frente a um opositor capaz de sustentar a regularidade proposta (GROPPEL, 1993; GROPPEL, 1992). Sendo esta a situação, a preparação (GP) de futuras situações de definição (GD) passa a ser fundamental (BALBINOTTI; MOTTA, 2009a; WOODS; FERNANDES, 2001; WOODS; HOCTOR; DESMOND, 1995).

É provável que estes conhecimentos básicos sobre a estrutura de desenvolvimento de uma competição no tênis sejam de alguma forma entendidos por uma parte da amostra, dada a sua experiência anterior. Não seria surpresa se, inclusive, fossem estes os conteúdos inseridos no planejamento de seus treinamentos técnicos.

Devemos ainda levar em consideração que a imensa maioria das quadras existentes em nosso estado, (e em nosso país) é feita de saibro. Este material torna o jogo mais lento, o que propicia trocas longas de bola durante os pontos (BALBINOTTI et. al, 2004a; WOODS; FERNANDES, 2001; CRESPO; MILEY, 1999). Também neutraliza significativamente o poder dos golpes vencedores (GD) pela diminuição da velocidade da bola. Estas são as principais razões que justificam os resultados revelados e explicam o porquê que os GP apresentaram maiores médias de freqüência de prática em relação aos GD.

Mas o que causou a maior surpresa foi a diferença significativa das médias obtidas entre a freqüência da prática de GP $(20,69)$ e GD $(15,87)$. Isto porque, apesar da importância da regularidade e da tentativa de pressionar o adversário a cometer erros, ainda constata-se que a cultura do tênis incentiva e a rigor valoriza a potência dos golpes. Provavelmente em razão do impacto positivo que esses golpes trazem em uma competição equilibrada (JONES, 1982).

A idéia dominante entre os apreciadores deste esporte é de que o jogo se resolve a priori a partir de iniciativas de finalização (GD) com forte incidência de erros. Tanto nas categorias juvenis quanto adultas federadas este princípio se reflete na tentativa de reproduzir performances observadas em jogadores profissionais (GRANITTO, 2001; WOODS; FERNANDES, 2001; GROPPEL, 1993). Aparentemente, o tênis no alto nível profissional é decidido pela impetuosidade e virtuosismo dos golpes indefensáveis dos atletas. Mas esta realidade se desfaz no momento em que realizamos qualquer análise sobre o andamento de partidas oficiais entre tenistas profissionais.

Um olhar mais atento sobre estas práticas revela que as ações que definem os pontos e são decisivas no resultado final são àquelas relacionadas com os Golpes de Preparação. Os pontos se decidem, na sua maioria, nos erros efetuados e não nas jogadas indefensáveis (McGRAW, 2002; CRESPO; MILEY, 1999; WOODS; 
HOCTOR; DESMOND, 1995; GROPPEL, 1993). Além disso, mesmo estas últimas necessitam de seqüências de golpes combinados que provoquem uma situação favorável a sua realização (GP). Nesse sentido, percebe-se claramente uma situação na qual, na tentativa de induzir o surgimento de um momento de definição, o que ocorre com maior freqüência é a falha do adversário (GRANITTO, 2001; WOODS; HOCTOR; DESMOND, 1995; GROPPEL, 1993; JONES, 1982).

O surgimento destes resultados preliminares indica uma clara mudança de rumo na condução dos treinamentos técnicos do tênis. Muitos instrutores e treinadores também comungam das idéias equivocadas apresentadas e incorrem em erros passíveis de serem evitados: estruturam seus planejamentos de treino sobre idéias muitas vezes copiadas de um modelo imaginário baseado em performances de tenistas profissionais que não condizem com a realidade. Além disso, priorizam seqüências de jogadas que buscam a finalização do ponto rapidamente e, muitas vezes, fora do contexto do jogo. Preferem estimular a prática sistemática de jogos formais, com contagem oficial (sets), ao invés de investirem num treinamento que exponha seus alunos a situações que claramente apresentem suas deficiências técnicas para, então, poderem ser corrigidas (McGRAW, 2002; VAN AKEN, 1999; WOODS; HOCTOR; DESMOND, 1995; GROPPEL, 1993; JONES, 1982). Tudo leva a crer que este equívoco está sendo corrigido, se levarmos em conta os resultados obtidos na amostra geral deste estudo.

Não se deseja aqui, de forma alguma, definir os GD como irrelevantes e de caráter menos importante para o desenvolvimento da performance dos tenistas adultos de competições federadas. Os GD têm a sua importância e em vários momentos devem ser utilizados. Apenas se pretende demonstrar que em várias situações de competição, os GP também são fundamentais pelo simples fato de que sem eles não existem GD bem colocados dentro do processo de finalização do ponto (BALBINOTTI; MOTTA, 2009a; CRESPO; MILEY, 1999).

Este fato nos oferece fortes indicativos de uma condução mais adequada do treino por parte dos sujeitos envolvidos neste processo, como treinadores e atletas. Os indícios apontam para uma mudança de mentalidade e quebra de paradigmas, pois o tênis vinha sendo entendido como um jogo basicamente de execução de golpes isolados em que as seqüências planejadas de técnicas combinadas normalmente apareciam em segundo plano, privilegiando-se a potência e a precisão dos golpes como definidores reais dos pontos e a prática de sets como melhor maneira de desenvolver a performance.

Ao controlarmos a variável "categoria", é possível indicar padrões que sugerem o motivo pelo qual tenistas da mesma faixa etária e semelhante experiência anterior na prática da modalidade participem de competições consideradas de nível técnico distintos. Já vimos que o volume de treino também é semelhante e não poderíamos tentar explicar este fenômeno seguindo por este caminho.

Resta-nos inferir sobre o conteúdo destas sessões de treino. Os resultados apontam no sentido de que para a dimensão GD, as três categorias ("Livre", "Maiores A" e "Maiores B") não apresentaram diferenças estatisticamente significativas entre si (sendo consideradas indissociáveis). Já no que diz respeito aos GP, as categorias "Livre" e "Maiores A" se mostraram indissociáveis e mais praticadas quanto à sua freqüência de treino do que a categoria "Maiores B". A este fato associam-se os

Pensar a Prática, Goiânia, v. 14, n. 2, p. 1-15, maio/ago. 2011 
10.5216/rpp.v14i2.10269

princípios já abordados sobre a importância dos GP no desenrolar eficaz de uma partida oficial de tênis. Os tenistas com possibilidade técnica de participar das categorias mais qualificadas ("Livre" e "Maiores A"), não por acaso, são aqueles que revelaram praticar com maior freqüência seqüências de GP, tornando-os mais aptos a solucionar os desafios técnicos inerentes ao jogo de tênis em seu favor (BALBINOTTI; MOTTA, 2009a).

Ao praticarem de forma mais freqüente os GP, valorizam mais a construção de jogadas, aumentam a regularidade dos golpes e diminuem a execução em momentos impróprios (BALBINOTTI; MOTTA, 2009a; GRANITTO, 2001; CRESPO; MILEY, 1999; DENT, 1995; FORTI, 1992). Ou seja, praticam seqüências técnicas mais eficazes e com menor incidência de erros.

O fato de haver associação entre as categorias "Livre" e "Maiores A" pode estar relacionada à grande semelhança de entendimento da técnica e do jogo de tênis entre estes dois grupos. De fato, a sua distinção em duas categorias de competições poderia estar relacionada com o volume e a intensidade de treinamento em momentos anteriores da carreira do tenista, principalmente na adolescência (BALBINOTTI; MOTTA, 2009a; BRECHBUHL; ANKER, 2000; UNIERZYSKI, 2007). É provável até que muitos dos tenistas pertencentes à categoria "Maiores A" participem eventualmente de competições próprias a categoria "Livre". A diferença técnica entre as categorias provavelmente se deve a maturidade já alcançada em alguns golpes por parte dos tenistas da categoria "Livre". Mas, ao incrementar a prática principalmente dos GP, esta diferença pode ser vencida e a progressão de categoria é uma possibilidade real a ser alcançada pelos tenistas da categoria "Maiores A" (BRABENEC; STOJAN, 1997).

No que tange à categoria "Maiores B", fica o indicativo de que um incremento da freqüência da prática de GP os aproximaria mais dos outros grupos que demonstram nível técnico mais elevado. Há indícios de que deve ser dada maior atenção a pratica de seqüências voltadas a regularidade e criação de situações que não tenham como prioridade a finalização rápida do ponto (WOODS; FERNANDES, 2001; BRABENEC, 1999; BRABENEC; STOJAN, 1997). Um entendimento mais realista de como o jogo se desenrola e se decide deve ser passado por parte dos treinadores dos atletas pertencentes a este grupo para que evitem a pressa da definição do ponto e prestigiem a técnica que contribua com a construção inteligente das jogadas.

Quanto à constatação de que não há diferenças estatisticamente significativas na freqüência da prática de GD para as três categorias oficiais, demonstram-se mais uma vez que não está na seqüência destes golpes a resposta para o sucesso no tênis. Se tenistas de diferentes categorias com distintos níveis técnicos praticam de forma muito semelhante os GD, não pode estar aí a explicação para o que lhes distingue.

É possível também especular que esta aproximação se dá muito mais por parte dos tenistas das categorias "Livre" e "Maiores A", pois além de estarem abrindo mão da prática dos GD, ainda estão optando em privilegiar os GP. Provavelmente por já entenderem que estes, de fato, são os mais importantes para o crescimento técnico e obtenção de resultados nas competições (BALBINOTTI; MOTTA, 2009a; BRECHBUHL; ANKER, 2000). Esta afirmação se baseia nas médias obtidas que

Pensar a Prática, Goiânia, v. 14, n. 2, p. 1-15, maio/ago. 2011 
10.5216/rpp.v14i2.10269

revelam uma diminuição extremamente significativa por parte destas duas categorias entre a freqüência de prática de GP e GD.

\section{Conclusões}

O presente estudo, para além de cumprir com as exigências teóricometodológicas de uma pesquisa científica, buscou com afinco dialogar - na medida do possível - com os treinadores de tenistas adultos. Para tanto, se tentou articular esta aproximação apresentando e discutindo os resultados de modo que possam servir de forma contundente e reveladora as novas tendências que norteiam o treinamento das técnicas avançadas no tênis. Nesse sentido, as principais conclusões que permitem apontar para novas perspectivas futuras foram as seguintes:

1) Os resultados obtidos na amostra geral indicam uma mudança relevante na maneira de conduzir o treinamento das técnicas avançadas: o treino, enfim, se aproxima de ações que ocorrem com frequência nas competições;

2) $\mathrm{O}$ fato da dimensão GP ser destaque na maioria das análises e comparações demonstra que essas ações são, indiscutivelmente, as mais relevantes para o incremento da performance competitiva de tenistas adultos federados;

3) O treinamento dos GP é prioritário para os tenistas de menor nível técnico (Maiores B), pois foram esses que apresentaram a menor frequência de treinamento dos GP;

4) E, por último, talvez a conclusão mais relevante desse estudo, foi a constatação de que a maior frequência no treinamento dos GP pode ser o diferencial na performance entre as três categorias que integram as competições oficiais da FGT.

\section{Referências}

ANGERS, M. Initiation pratique à la méthodo logie des sciences humanies. Montreal: Les Éditions de ls Cheneliére, 1992.

ASÇI,F.H.; ASÇI, A.; ZORBA, E. Cross-cultural validity and reliability of phisical selfperception profile. International Journal of Sports Psychology. vol.3, Rome, 1999.

BALBINOTTI, C.A.A. A formação técnica do jogador de tênis: um estudo sobre jovens tenistas brasileiros. Tese de doutorado, Porto: Universidade do Porto, 2003a.

BALBINOTTI, M.A.A. Para se avaliar o que se espera: reflexões acerca da validade dos testes psicológicos. Aletheia, Canoas, v.21, n.1, p.43-52, 2005.

BALBINOTTI, M.A.A.; BALBINOTTI, C.A.A.; MARQUES, A.T.; GAYA, A.C.A Estudo descritivo do "Inventário do Treino Técnico-desportivo do Tenista": resultados parciais segundo o sexo. Revista Portuguesa de Ciências do Desporto, Porto, v.3, n.3, p.7-17, jul-dez, 2003b. 
10.5216/rpp.v14i2.10269

BALBINOTTI, M.A.A.; BALBINOTTI, C.A.A.; MARQUES, A.T.; GAYA, A.C.A. O treino técnico-desportivo de jovens tenistas brasileiros. Revista Brasileira de Ciências do Esporte, Campinas, v.26, n.1, p.51-72, set., 2004a.

BALBINOTTI, M.A.A.; BALBINOTTI, C.A.A.; MARQUES, A.T.; GAYA, A.C.A Proposição e validação de um instrumento para avaliação do treino técnico-desportivo de jovens tenistas. Revista Brasileira de Educação Física e Esporte, São Paulo, v.18, n.3, p.213-226, julho/set., 2004b.

BALBINOTTI, M.A.A.; BALBINOTTI, C.A.A.; MARQUES, A.T.; GAYA, A.C.A Estudo descritivo do Inventário do Treino Técnico Desportivo: resultados parciais segundo o 'ranking'. Revista Portuguesa de Ciências do Desporto, Porto, v.5, n.1, p.49-58, 2005.

BALBINOTTI, C.; BALBINOTTI, M.; MARQUES, A.; GAYA, A. O treino técnico desportivo do tenista infanto-juvenil (13 a 16) anos: um estudo descritivo-exploratório com três grupos submetidos a diferentes cargas horárias de treinos semanais. Revista Brasileira de Ciências do Esporte. Florianópolis: v. 29, 2008.

BALBINOTTI, C; MOTTA, M. A Bola em Jogo no Tênis: O Domínio das Técnicas Avançadas. In: O Ensino do Tênis: novas perspectivas de aprendizagem. Porto Alegre: Artmed, $2009^{\text {a }}$

BALBINOTTI, C; MOTTA, M. Os Modelos Estratégico-táticos do Tênis de Competição. In: O Ensino do Tênis: novas perspectivas de aprendizagem. Porto Alegre: Artmed, 2009 b.

BARTHOLOMEW, J.B.; EDWARDS, S.M.; BREWWER, W.; RAALTE, J; LINDER, D.E. The sports inventory for pain: a confirmatory factor analysis. Research Quartely for Exercise and Sports. Washington: vol. 1, 1998.

BISQUERA, R. Introdución a La estadística aplicada a La investigción educative: um enfoque informático com los paquetes BMDP y SPSS. PPU. Barcelona: 1987.

BRABENEC,J ; STOJAN, S. Gran Jugador o Sólo un Buen Jugador? ITF Coaches Review. Londres: n. 12; 1997.

BRABENENEC, J. La Competición: la forma de entrenamiento más deseable. ITF Coaches Review. Londres: n. 17, 1999.

BRECHBUHL, J.; ANKER, P. El Método de Acción en el Tennis. ITF Coaches Review. Londres: n. 22, 2000.

BRYMAN, A.; CRAMER, D. Quantitative data analysis with SPSS release 8 for Windows: a guide for social scientists. New York: Routledge, 1999. 
CRESPO, M.; MILEY, D. Manual Para Entrenadores Avanzados. Londres: International Tennis Federation (ITF), 1999.

DELFORGE, C. Analysis of player-parent relationships and the role of the coach. ITF Coaching and Sports Science Review. v. 14, n. 38, London: 2006.

DENIAU, G. Tenis: técnica, táctica y entrenamiento. Barcelona: Paidotribo, 1991.

DENT, P. Awareness of standars. ITF Coaches Review. Londres: n. 07, 1995.

EKLUND, R.; WHITEHEAD, J.; WELK, G. Vality of the childrean and youth physical self-perception profile: a confirmatory analysis. Research Quarterly for Exercise and Sports. Washington:vol. 3, 1997.

FORTI, U. Curso Avanzado de Tênis. Barcelona: Ed. De Vechi, 1992.

GRANITTO, G. Qué tipo de inteligência pueden tener los jugadores exitosos? ITF Coaches Review. Londres: n. 23, 2001.

GROPPEL, J. Tenis para jugadores avanzados. Madrid: Gymnos, Editorial Desportiva, 1993.

JONES, C. M. Tácticas de tênis: analisis del jugador y del juego para vencer. Barcelona: Editora Hispano Europea, 1982.

MAGUIRE, T.; ROGERS W. Proposed solutions for non randomness in educational research. Canadian Journal of Education, V. 14, n. 2, p. 170-181, 1989.

McGRAW, P. Filosofia del desarrollo del jugador. ITF Coaches Review. Londres: $n$. $28,2002$.

PESTANA, M. H.; GAGEIRO, J. G. Análise de dados para ciências sociais: a complementaridade do SPSS (3 ${ }^{\mathrm{a}}$ Ed.). Lisboa: Edições Silabo, 2003.

REIS, E. Estatística descritiva. Lisboa: Edições Silabo, 2000.

SCHÖMBORN, R. Tênis: entrenamiento técnico. Madrid: Ed. Tutor, 1999.

SIRKIN, R. M. Statistics for the Social Sciences. London: Sage Publications, 1999.

THOMAS, J.; NELSON, J. Métodos de Pesquisa em Atividades Físicas. $3^{\text {a }}$ Ed. Porto Alegre: Artmed, 2002. 
10.5216/rpp.v14i2.10269

UNIERZYSKI, P. ; CRESPO, M. Review of modern teaching methods for tennis. International Journal of Sport Science. Madrid: v. 3, 2007.

VAN AKEN, I. El processo de aprendizaje táctico e técnico. ITF Coaches Review. Londres: n. 19, 1999.

WOODS, R.; FERNANDEZ, M. Finding the best learning style. IN: Word- class Tennis Technique. Roetert, P.; Groppel, J. (Eds.). Champaing: Human Kinetics, 2001.

WOODS, R.; HOCTOR, M.; DESMOND, R. Coaching tennis successfully. United States Tennis Association. Champaign: Human Kinetics, 1995.

ZLESAK, F. Preparación de jugadores juniors para el tênis professional. Publicaciónes Real Federación Espanola de Tênis. 1994a.

ZLESAK, F. Como mejorar la técnica (de nivel básico e avanzado). Publicaciónes Real Federación Espanola de Tênis. 1994b.

\section{THE TRAINING OF ADVANCED TECHNIQUES OF FEDERATED ADULT TENNIS PLAYERS: A COMPARATIVE STUDY AMONG THREE DIFFERENT CATEGORIES}

Abstract: The training of advanced techniques in tennis consists in the systematic training of Preparation Strokes (GP) and Definition Strokes (GD). These are combinations of strokes that are very executed during the competitions. The aim of this study is to answer two questions: (1) What is the frequency of training of the Preparation Strokes and the Definition Strokes of federated adult players?; (2) Are there significant differences when comparing the results of the three official categories established by the "Gaúcha Tennis Federation" (FGT): "Livre", "Maiores A" and "Maiores B"? In this verification was applied the Inventory Training of the Tennis player (ITTT-12), in which the players answered how often they train the top 12 playing actions that make up the advanced techniques in tennis. With the results obtained, we concluded that the GP are, no doubt, the most relevant actions for the performance of federated adult players.

Keywords: Tennis. Advanced Techniques. Adult Tennis Players.

\section{LA FORMACIÓN DE TÉCNICAS AVANZADAS DE TENIS ADULTOS FEDERADOS: UN ESTUDIO COMPARATIVO ENTRE TRES CATEGORÍAS DIFERENTES.}

Resumen: El entrenamiento de técnicas avanzadas en el tenis es el ejercicio sistemático de las estafas de preparación (GP) y los contras Definición (GD). Estas son las combinaciones de golpes efectuados durante la competición. El objetivo de este estudio es responder a dos preguntas: (1) ¿Cuál es la frecuencia del entrenamiento y la 
10.5216/rpp.v14i2.10269

preparación de las estafas Estafas definiciones de los jugadores federados de tenis para adultos, (2) Existen diferencias significativas en la comparación de los resultados de las tres categorías oficiales establecidas por la Federación Tenis Gaucho (FGT): "La libertad, el Mayor A, B, el mayor"? Con este fin, se aplicó el Inventario de los deportes de Formación Técnica del tenis (ittt-12), en los que los jugadores responden con frecuencia con la que formar a los mejores 12 poblaciones que conforman las técnicas avanzadas en el tenis. A través de los resultados, llegamos a la conclusión de que el GP es, sin duda las acciones más importantes en el desempeño de los jugadores adultos federales de tenis.

Palabras clave: Tenis. Técnicas Avanzadas. Tenis de Adultos.

Endereço para correspondência:

cbalbinotti@terra.com.br

Marcos Alencar Abaide Balbinotti

Universidade Federal do Rio Grande do Sul

Rua Felizardo, 750

Jardim Botânico

90690-200 - Porto Alegre, RS - Brasil

Pensar a Prática, Goiânia, v. 14, n. 2, p. 1-15, maio/ago. 2011 